\title{
Object-based apparent motion
}

\author{
ASHER KORIAT \\ University of Haifa, Haifa, Israel
}

\begin{abstract}
The interpretation of a dynamic visual scene requires integrating information within frames (grouping and completion) and across frames (correspondence matching). Fragmentary views of objects were used in five experiments. These views could not be matched with each other by any similarity transformation on the basis of their explicit visual features, but their completed versions were related by a rotational transformation. When the fragmentary images were successively presented to observers, it was found that they produced apparent motion in the picture plane and in depth. Thus, apparent motion is capable of establishing correspondence at the level of perceptually recovered objects in three-dimensional space.
\end{abstract}

The primary goal of the visual system is to construct a representation of distal objects in the external world. When confronted with a stationary scene, the visual system must integrate the information that is available in the image in order to form a representation of separate coherent objects. When the scene is dynamic, the visual system must also integrate information across different time slots (or "frames") to construct a more complex representation, in which several objects may be moving in different orbits, while others remain stationary. Both forms of integration, within and between frames, encounter the difficulty that some of the visual features are often missing from the image(s). Because depth information is lost in the projection from the environment onto the retina, and some of the information may also be missing because of accidental occlusion and visual noise, some aspects of the scene must be inferred in constructing a distal representation from a proximal projection (see Nakayama, Shimojo, \& Silverman, 1989).

The present study concerns two phenomena that reveal the way that the visual system makes such inferencesperceptual grouping and apparent motion. These disclose the operation of two mechanisms that are designed to exploit regularities in the proximal visual array to fill in some of the missing links. In perceptual grouping, some organization is imposed on an image - visual segments that presumably belong to the same object are assembled, and connectivity and coherence are restored. In apparent motion, on the other hand, the correspondence

\footnotetext{
The experiments were conducted at the Institute of Information Processing and Decision Making, University of Haifa. This paper was completed while the author was the Irv Acenberg visiting scientist at the Rotman Research Institute of Baycrest Centre, Toronto. I want to thank Marlene Behrmann, Irving Biederman, Nancy Kanwisher, Ennio Mingolla, Romi Nijhawan, Joel Norman, and an anonymous reviewer for their valuable comments on an earlier draft. I also thank Shiri Avneon for help in conducting the experiments, and Hanna Strominger for programming the experiments. Requests for reprints should be addressed to A. Koriat, Department of Psychology, University of Haifa, Haifa, Israel.
}

between two successive images results in the subjective experience that they depict two phases in the history of the same object as it is undergoing transformation. In the present study, the relationship between these two mechanisms was examined through an investigation of the nature of the representation underlying apparent motion.

Perceptual grouping must solve the problem of what features belong together, which is particularly critical when there are several occluding objects and when some of the object contours are missing. Thus, in extracting a three-dimensional (3-D) distal representation, some of the missing information in the proximal 2-D image itself must be inferred and filled in through a process of completion or restoration. Biederman demonstrated that object recognition can be effective even when a large proportion of the contours is deleted (Biederman, 1987; Biederman \& Cooper, 1991b; Blickle, 1989). According to his recognition-by-components model (Biederman, 1987; Hummel \& Biederman, 1992), objects can be recognized on the basis of a small number of volumetric primitives, called geons, which can be directly extracted from the 2-D image independently of viewing position. Because of the redundancy in their image features, geons can be readily identified under moderate degrees of degradation and occlusion, when only part of their visual features are available.

Perceptual grouping and contour restoration may be guided by Gestalt principles, such as good continuation, closure, and symmetry (Koffka, 1935), which operate on the edge information itself (Grossberg \& Mingolla, 1985). Apparently, these processes takes time (Kellman \& Shipley, 1991; Sekuler \& Palmer, 1992; Snodgrass \& Feenan, 1990). Biederman observed that when the missing visual features could be readily recovered through routines of collinearity or curvature that restore contours, object recognition was much easier than it was when deletion destroyed recoverability. Nevertheless, at brief exposure durations, deleted recoverable versions of an object took longer to recognize than intact versions, suggesting again that the completion process takes time, 
and it is needed for shape recognition (Biederman, 1987; Blickle, 1989).

Perceptual grouping and closure operate to fill in discontinuities in space within a single visual frame, but apparent motion interpolates discontinuities over time by establishing correspondence between successive visual frames. Under suitable conditions, alternating static images that are related by a shape-preserving similarity transformation produce the perception of a rigid motion over a specific trajectory. A minimum temporal separation between the two images is required for such motion, and this minimum increases linearly as a function of the spatial separation between them (Bundesen, Larsen, \& Farrell, 1983; Farrell, 1983; Farrell, Larsen, \& Bundesen, 1982; Farrell \& Shepard, 1981; Korte, 1915; Shepard \& Judd, 1976). It is generally assumed that the intercept of this function measures the time it takes to encode the two successive stimuli as different views of the same object, and that the slope reflects the maximum speed of interpolating a path between them (McBeath \& Shepard, 1989; Shepard, 1984).

Apparent motion must solve the correspondence problem of identifying the matching elements in the two views (Ullman, 1979). There is debate, however, as to whether correspondence matching is a low-level process that matches elements point-for-point according to their distances in the 2-D projection (Anstis, 1970; Mutch, Smith, \& Yonas, 1983; Ullman, 1978, 1979), or whether it represents a high-level process that is based on global correspondence between objects as they are interpreted in 3-D space (Attneave \& Block, 1973; Green \& Odom, 1986; Shepard \& Cooper, 1982; Shepard \& Judd, 1976). A related issue is whether apparent motion precedes the extraction of form or follows such extraction (Petersik, 1989). A resolution of the conflicting views has been proposed. This resolution is based on the distinction between short- and long-range processes in apparent motion, but some of the issues pertaining to this distinction are not yet settled (Braddick, 1980; Cavanagh \& Mather, 1989; Petersik, 1989; Shepard \& Cooper, 1982).

In the present study, the representation underlying apparent motion was examined by the investigation of apparent rotational motion between contour-deleted views of objects. Assuming that the visual system must integrate information both within and across visual frames, the question, then, is whether the cross-frame correspondence matching can be performed on the outcome of the within-frame completion process. If correspondence matching can be performed at the level of object representations, then, under some conditions, apparent motion may be expected to take place at the level of the restored objects, between two fragmentary views that cannot be directly matched by any similarity transformation.

Indeed, previous work suggests that apparent motion is modulated by an earlier stage of figure-ground segregation (see Ramachandran \& Anstis, 1986), even when such segregation depends on occlusion cues involving "modal" (occluding) or "amodal" (occluded) subjective contours (see Kanizsa, 1979). Thus, apparent motion can be obtained between illusory shapes: When two figures are presented in succession, so that the illusory shape occupies different positions in the two figures, good apparent motion is generally reported (Mather, 1988; Ramachandran, Rao, \& Vidyasagar, 1973). Apparent motion has also been reported in cases in which an "invisible" object is seen to be moving behind a larger occluding object (Anstis \& Ramachandran, 1985). Several observations suggest that the correspondence process underlying apparent motion is also influenced by the completion of "amodal," occluded contours (Shimojo \& Nakayama, 1990). Although these observations suggest a high-level motion process in which the extraction of form cues precedes motion detection, there are indications that apparent motion, in itself, can help segment a scene into coherent visual entities (e.g., Mather, 1988; Nakayama et al., 1989; Shimojo, Silverman, \& Nakayama, 1989) and generate the perception of occlusion (e.g., Anstis \& Ramachandran, 1985).

In the present study, the relationship between form extraction and motion detection was investigated with the use of pairs of complementary, contour-deleted images, similar to those employed by Biederman and colleagues (Biederman \& Cooper, 1991b; Blickle, 1989). It was hoped that this approach would help to tie the study of apparent motion to some of the current issues in object recognition (e.g., Hummel \& Biederman, 1992; Srinivas, 1993). In the complementary pairs, each image depicted a fragmentary view of the same object, from which the full object could be readily recovered through processes of completion. Note that the missing contours in these images (see Figure 1) are like those produced by occluding surfaces, but there are few depth or form cues that could allow the demarcation and segregation of a coherent occluding shape (see, e.g., Shimojo \& Nakayama, 1990). The two complementary images were generated so that the line segments deleted from one image would be retained in the other image, and vice versa. Thus, if it is found that the alternating presentation of the two images in different orientations yields apparent rotational motion of the depicted object, this motion could not be explained on the basis of a partial or complete point-to-point matching between the two images (see Cavanagh \& Mather, 1989; Ramachandran, 1990).

A rotational transformation was deliberately chosen in the present study, because it has been found to be particularly sensitive to the figural properties of the stimulus (e.g., Farrell et al., 1982; Farrell \& Shepard, 1981; Shepard \& Judd, 1976). Two successively presented shapes can give rise to the phenomenal experience of a single object in motion when they are related by a shapepreserving transformation (Warren, 1977; but see Pittenger \& Shaw, 1977). Even when the two shapes are nonalignable in 2-D space, they can be experienced as two points in the history of a single object when the visual system specifies their shape-preserving transformability in 3-D space (e.g., Shepard \& Judd, 1976). Rotational apparent motion, then, appears to be geared 
to the extraction of shape-invariant properties of objects. Note, however, that a plastic, nonrigid motion can also be perceived between two shapes that are not transformable. This occurs particularly in the most commonly studied type of apparent motion-translation through space. Thus, an object can appear to change its shape as it moves, or even "change into" a different object. These observations have led some researchers to argue that shape makes little contribution to apparent motion (see, e.g., Kolers, 1972; Kolers \& Pomerantz, 1971; Victor \& Conte, 1990; but see Warren, 1977; Warren \& Shaw, 1978).

The present study does not bring into question the possibility that apparent motion can be based on local correspondence and can occur between objects with different shapes. Rather, it examines the question of whether apparent motion can also occur at the level of object representations when it is not supported (but also not counteracted) by local-level correspondence. Accordingly, the experiments were deliberately designed to seek out those conditions that would be beneficial for the occurrence of object-based apparent motion. Line drawings of simple geometric shapes (e.g., a rectangle) were used as "objects." In creating two complementary images for each such object, an attempt was made (1) to assure that the object would be recoverable from each of its two images (but see Experiment 5), and (2) to minimize the possibility of local-level matches that would conflict with alignment at the object level.

The general methodology of Experiments 1-4 was as follows. On each trial, two views of the same object were presented in rapid alternation, for a total of eight frames. The stimulus onset asynchrony (SOA) was initially set at a value, $\mathrm{SOA}_{\mathrm{i}}$, that was too short to give rise to smooth apparent rotational motion between the two images. Each right keypress increased SOA by $10 \%$ of $\mathrm{SOA}_{p}-\left(\mathrm{SOA}_{i}-35\right)$ msec, where $\mathrm{SOA}_{\mathrm{p}}$ was the previously presented SOA. A left keypress reduced it by the same amount. The subjects were instructed to increase
SOA gradually until a smooth rigid motion was perceived between the two views. Then they terminated the trial, and the final SOA value was recorded. They were instructed not to proceed beyond the point at which a smooth rigid motion was perceived. If they did so, they were to speed up the rate of alternation until the motion was destroyed, and try again. The dependent variable was the minimum SOA required for rigid apparent motion.

\section{EXPERIMENT 1 \\ Apparent Rotation in the Frontal Plane}

Previous experiments, in which the same shape was alternately presented at different orientations, have established that the minimum SOA for apparent shapepreserving rotational motion increases linearly with the angular separation between the two stimuli (Farrell, 1983; Farrell et al., 1982; Shepard \& Judd, 1976). In Experiment 1 , a full (intact) rectangle was used, together with two fragmentary versions, the sides and corners versions (see top row of Figure 1). The latter two versions represented complementary images that created a rectangle when properly superimposed on each other. For example, on some trials, the subjects saw the sides and corners images in alternation, so that one of these appeared at the "upright" orientation (top row of Figure 1), while the other appeared rotated $40^{\circ}, 60^{\circ}$, or $80^{\circ}$ to the right or to the left (for an example, see the bottom row of Figure 1). Note that the angular distance between the two complementary rectangles was defined as the (shortest) angular deviation between their presumed completed versions.

The first aim of the experiment was to determine whether a rigid apparent motion can be produced by the sequential alternation of two visually incongruent images of the same object, and, if so, whether it exhibits the same type of relationship to angular distance that was found for a repetition of the same exact stimulus at

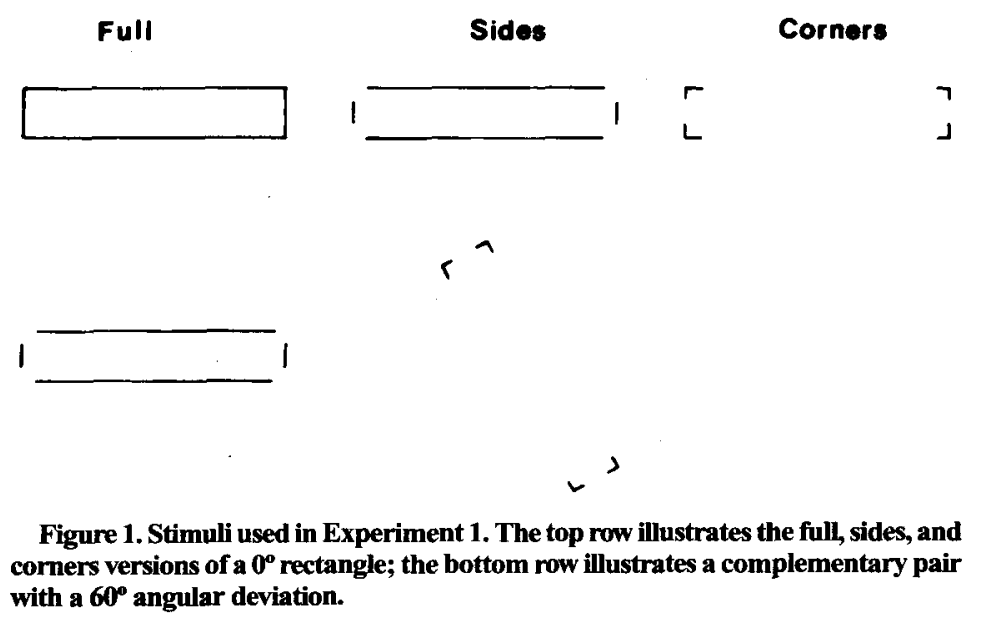


different orientations. The second aim was to explore the possibility that the encoding of two complementary figures as depicting the same object takes time. Therefore, the intercept of the function relating minimum SOA to angular deviation should be higher for alternating complementary images than for alternating identical images. This prediction is based on findings suggesting that the recognition of contour-deleted or contour-occluded objects requires more time than the recognition of their corresponding intact versions (Biederman, 1987; Blickle, 1989; Sekuler \& Palmer, 1992). To examine this possibility, additional conditions were included, in which two identical versions (i.e., both full, both corners, or both sides) were alternately presented.

\section{Method}

Apparatus and Stimuli. All the experiments were conducted on a computerized graphics display unit (Apollo Domain 4000). The stimuli appeared as black lines on a light-blue background, centered and rotated about the center of the screen. The stimuli for Experiment 1 were three images of a rectangle - full, sides, and corners-as illustrated in the top row of Figure 1. Each rectangle subtended $1 \times 5 \mathrm{~cm}$ (visual angles: $0.7^{\circ} \times 3.6^{\circ}$ ).

Procedure. The subjects viewed the screen at a distance of $80 \mathrm{~cm}$, so that the longest side of the rectangle subtended a visual angle of approximately $3.6^{\circ}$. Two images of the rectangle were used on each trial; they were displayed in sequential alternation at different orientations on the computer terminal. There were five types of pairs: full-full (FF), corners-corners (CC), sides-sides (SS), corners-sides (CS), and sides-corners (SC). Odd-numbered frames always consisted of horizontal $\left(0^{\circ}\right)$ displays (as in the top row of Figure 1); in the remaining frames, the rectangle appeared rotated $40^{\circ}, 60^{\circ}$, or $80^{\circ}$ clockwise or counterclockwise. Thus, for an SC pair, for example, the sequence was SCSCSCSC, with all "sides" stimuli horizontal. The eight frames were separated by blank interstimulus intervals of $45 \mathrm{msec}$. The bottom row of Figure 1 illustrates a complementary (SC) pair with a $60^{\circ}$ angular deviation.

Each pairing of two images appeared in six different combinations of orientations (defined by the signed angular deviation between them), yielding a block of 30 different sequences. These sequences were presented in a random order, except that each group of five successive trials included all five pair types, and that none of the pair types were repeated in immediate succession. Two blocks were administered in each of three sessions, which took place on different days (no more than 2 days apart). The first session also included a practice block, in which 5 -cm-long lines were used instead of rectangles.

On each trial, frame duration was initiated at $80 \mathrm{msec}$, thus setting SOA at $125 \mathrm{msec}$. The subjects could increase SOA by increasing stimulus duration, while interframe interval remained constant throughout the experiment. ${ }^{1}$ They were told to repeatedly press the right-side key until they could perceive a continuous toand-fro rigid rotational motion between the two shapes. The limiting value of SOA was recorded by pressing the Y key (for "yes"), which also terminated the trial. The subjects were instructed not to proceed beyond the point at which a smooth apparent motion was perceived. If they did so, they were to speed up the rate of alternation (by pressing a left-side key) until the rigid rotational motion was destroyed, and try again. In this manner, the limiting SOA value was always attained by slowing down (rather than by speeding up) the alternation rate. The subjects were free to review the sequence of stimuli at any stage by pressing the space bar.

Subjects. Ten University of Haifa students were paid for participating in the experiment. They were naive as to the purpose of the experiment.

\section{Results}

Figure 2 presents mean minimum SOA as a function of angular deviation for the five sequence types. It can be seen that minimum SOA increased with angular deviation for all pairs, but identical pairs (FF, CC, and SS) evidenced shorter SOAs than complementary pairs (CS and SC). A two-way analysis of variance (ANOVA), sequence type (identical vs. complementary) $\times$ angular deviation (3), yielded $F(1,9)=12.31, p<.01$ for sequence type; $F(2,18)=17.53, p<.0001$ for angular deviation; and $F(2,18)=3.45, p<.10$ for the interaction. The effects of angular deviation were significant for both sequences, [identical, $F(2,18)=13.96, p<.0005$; complementary, $F(2,18)=12.17, p<.0005]$. The linear function relating minimum SOA to angular deviation had an intercept of 122.6 and a slope of 0.59 for the identical pairs. The respective values for the complementary pairs were 160.4 and 0.40 .

\section{Discussion}

The results of Experiment 1 indicate that shapepreserving apparent motion can transpire between two complementary images of the same object, and that this motion evidences a relationship between SOA and angular deviation that is the same as that found for the presentation of two identical images. These results suggest that apparent motion can establish correspondence at the level of the object representation. However, the results also disclose a higher intercept for complementary than for identical pairs, which is consistent with the idea that perceptual completion takes time (Biederman, 1987;

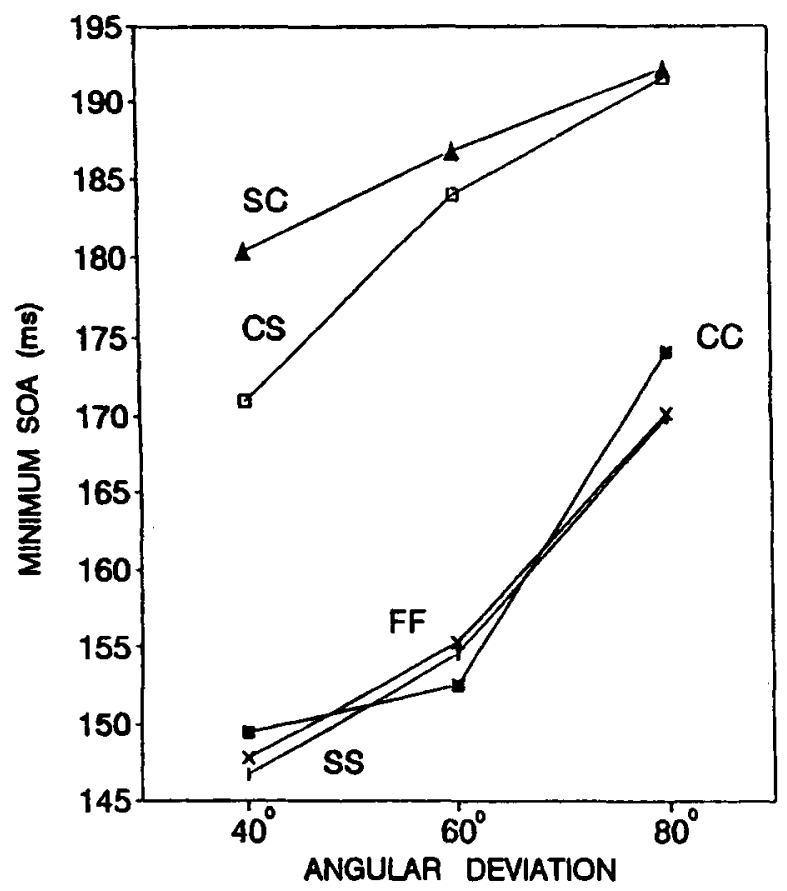

Figure 2. Mean minimum stimulus onset asynchrony as a function of angular deviation for the five pair types used in Experiment 1 (F, full; $\mathrm{S}$, sides; $\mathrm{C}$, corners). 
Kellman \& Shipley, 1991; Sekuler \& Palmer, 1992; Snodgrass \& Feenan, 1990).

The distinction between image-based and object-based apparent motion parallels the distinction between firstorder and second-order motion (Cavanagh \& Mather, 1989). First-order motion is assumed to respond to color and luminance, but second-order motion is said to be sensitive to differences in stimulus attributes such as spatial structure, movement, and disparity, even in the absence of first-order differences. According to Cavanagh and Mather, similar comparator mechanisms underlie both types of motion perception. The observation in Experiment 1 , which indicated that apparent motion between complementary images yields a relationship between SOA and angular deviation that is the same as that found for intact or complete images (e.g., Farrell et al., 1982; Shepard \& Judd, 1976), is consistent with this view. The results also suggest that image-based and object-based apparent motion share the same mode of operation.

The higher intercept obtained for complementary than for identical pairs suggests, perhaps, that correspondence matching may be achieved either at the image level or at the object level. Presumably, motion detection takes more time when it can be achieved only at the level of the recovered object than when it can be based directly on image features as well.

\section{EXPERIMENT 2 Separating Objects in Apparent Motion}

Experiment 2 addressed the question of whether the type of object-based apparent motion observed in the previous experiment can also be used to establish correspondence for two separate objects recovered from the same dynamic scene. As noted earlier, a fundamental problem facing the visual system is how to group together contours that presumably belong to the same object while segregating the visual scene into separate entities. Each of the fragmentary stimuli presented in the bottom row of Figure 3 contains sufficient cues to sup- port the recovery of two separate objects (or geons) - a rectangle and a triangle. The question is whether apparent motion would preserve this grouping, thus supporting the segregation of a dynamic scene into separately moving objects. Note that there is no simple global transformation that can bring the two images into alignment with each other; the same holds true for their respective constituent parts (triangles and rectangles). Therefore, object-based apparent motion would entail different interpolating paths for each of the two objects. Note also that a close scrutiny of the stimuli (Figure 3 ) is needed to infer the type of transformation required to bring the corresponding objects into alignment.

\section{Method}

Stimuli and Procedure. The stimuli are depicted in Figure 3. They form two pairs: a full pair, depicting intact triangles and rectangles, and a complementary pair, consisting of fragmentary versions of these shapes. The experiment included four trials with the full pair and four trials with the complementary pair. The two trial types were alternated, and choice of leading stimulus (A or B) was counterbalanced. Interframe interval was $60 \mathrm{msec}$ throughout, and SOA was initialized at $125 \mathrm{msec}$.

Subjects. Ten University of Haifa students were paid for participating in the experiment. None had participated in Experiment 1 .

\section{Results and Discussion}

It was found that the full and complementary pairs both produced an apparent motion that was described by some of the subjects as a "scissors motion." Minimum SOA averaged $275.6 \mathrm{msec}(S E=34.8 \mathrm{msec})$ for the complementary pair and $205.5 \mathrm{msec}(S E=21.7 \mathrm{msec})$ for the full pair $[F(1,9)=6.27, p<.05]$. These results suggest that object-based apparent motion can establish continuity for two separate objects, each moving in its own separate orbit.

Taken together, the results of Experiments 1 and 2 support the idea that, under some conditions, crossframe matching can be performed on the outcome of the
Full
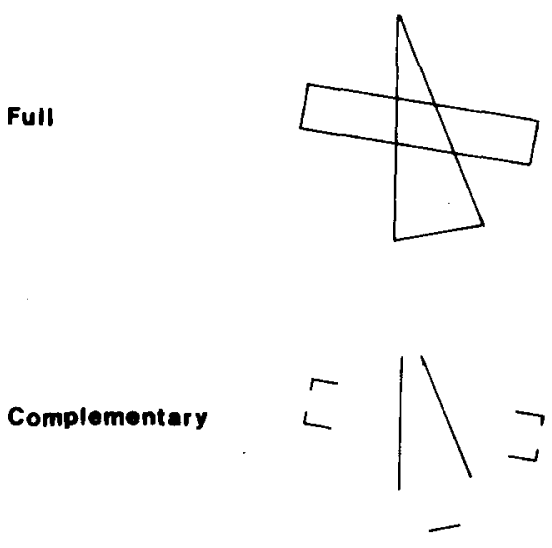
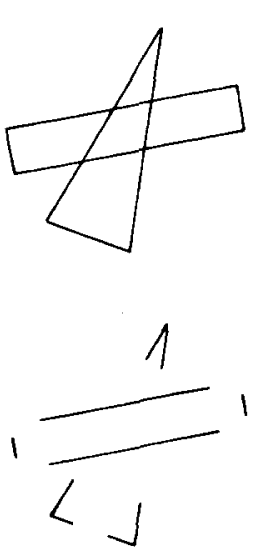

\section{A}

Figure 3. The full and complementary versions of the two stimuli (A and B) used in Experiment 2. 
within-frame grouping. Thus, it seems that apparent motion preserves the integration of visual features that belong to the same object, as well as the segregation of a scene into separate objects.

\section{EXPERIMENT 3 Rotation of Planar Objects in the Depth Plane}

In Experiment 3, the rotation of two surfaces in depth was examined. Previous work, in which two views of the same object were used, indicated that the minimum SOA required for apparent motion is nearly equal for distances in the picture plane and in depth. This has been shown for rectilinear motions (Corbin, 1942; Attneave \& Block, 1973), as well as for rotational motions (Shepard \& Judd, 1976). Also, when a stimulus has two neighbors of different apparent depths but similar 2-D distances, objects appear to move toward the neighbor with the same apparent depth (Green \& Odom, 1986). The results from experiments in which the effects of occlusion and shading cues on apparent motion were examined also suggest that apparent motion can occur after a 3-D representation is formed (Anstis \& Ramachandran, 1985; Kleffner \& Ramachandran, 1992; Shimojo \& Nakayama, 1990). The question that was addressed in Experiment 3 was whether apparent motion can establish correspondence at the level of the object representation in 3-D space when the two perspective views represent complementary images of the same object. Note that, here, complementarity must be defined at the level of the 3-D object, as will be explained shortly.

Consider the pairs of complementary figures depicted in Figure 4. These images are generally interpreted as perspective drawings that portray the same planar object (e.g., a floor-like surface). Although the members of each pair cannot be mapped onto each other by any similarity transformation, they are complementary at the object level; when properly rotated in depth, their superimposition produces a "full," or intact, image. Would such stimuli produce apparent motion in depth when displayed in alternation?

\section{Method}

Stimuli. The stimuli depicted in Figure 4 illustrate the complementary perspective views used in the experiment. The two images in the top row are related by a $20^{\circ}$ rotation in depth; those at the bottom are related by a $40^{\circ}$ rotation in depth. They were created by first rotating a square around the $x$-axis to produce a slant-indepth view; then the slanted square was rotated either $10^{\circ}$ or $20^{\circ}$ clockwise or counterclockwise around its center. The longest side of the $20^{\circ}$ image (bottom row) subtended $6.8 \mathrm{~cm}$ on the screen.

Three versions were prepared for each view: full (not shown in the figure), sides, and corners.

Procedure. On each trial, either a $20^{\circ}$ pair or a $40^{\circ}$ pair was presented, using the five sequence types that were defined in Experiment 1: FF, CC, SS, CS, and SC. The procedure and instructions were similar to those of Experiment 1. The experiment was conducted in two sessions that took place on 2 different days, no more than 2 days apart. Three blocks were presented in each session, with a 5-min break between the blocks. Each block consisted of 10 trials, which represented all combinations of sequence type (5) $X$ angular deviation (2), randomly ordered. The leading stimulus in a sequence (rotated clockwise or counterclockwise) was determined at random. The first session also included 10 practice trials -6 consisting of FF sequences - followed by one pair each of the remaining sequence types.

The subjects viewed the screen at a distance of $80 \mathrm{~cm}$, so that the longest side of the $20^{\circ}$ quadrangle subtended a visual angle of 4. $9^{\circ}$. Interframe interval was $100 \mathrm{msec}$, and stimulus duration was initialized at $60 \mathrm{msec}$.

Subjects. Ten University of Haifa students were paid for participating in the experiment. They were naive as to the purpose of the experiment, and none had participated in any of the previous experiments.

\section{Results}

The results for the four sequence types suggested that SOA increased with increasing angular deviation; they were somewhat shorter for identical pairs than for complementary pairs (see Figure 5). A two-way ANOVA, however, yielded $F(4,36)=1.46$, n.s. for pair type; $F<1$ for angular deviation; and $F<1$ for the interaction.
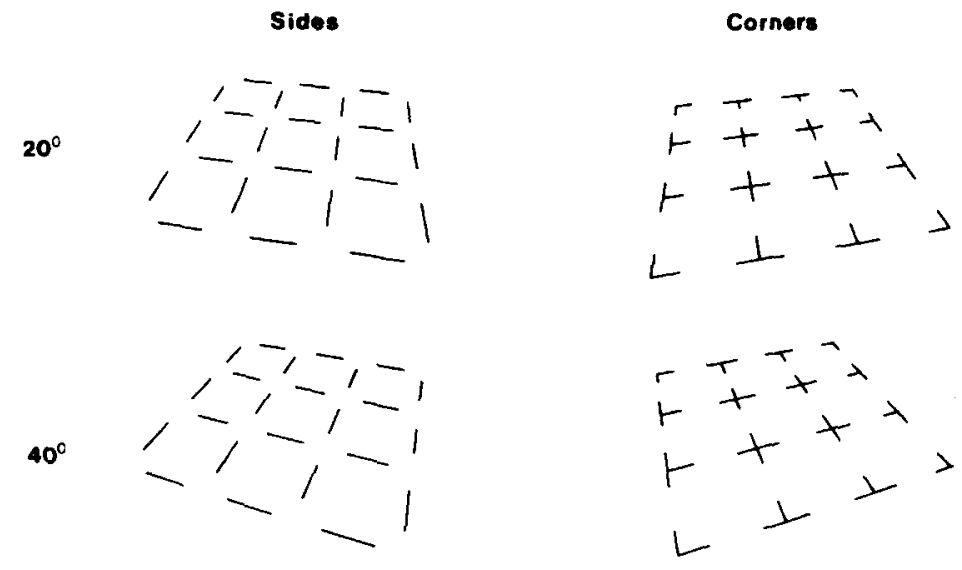

Figure 4. Pairs of complementary perspective views representing $20^{\circ}$ and $40^{\circ}$ angular deviations (Experiment 3). 
When the stimulus sequences were collapsed into identical (FF, CC, and SS) and complementary sequences (CS and SC), a two-way ANOVA yielded $F(1,9)=2.44$, n.s. for sequence type; $F<1$ for angular deviation; and $F<1$ for the interaction.

\section{Discussion}

The experiment failed to yield an effect of angular deviation, possibly because of the small range of deviations sampled. However, a smooth apparent motion was obtained even with the complementary images, showing SOAs that were only slightly longer than those of the identical images. In fact, an informal survey indicated that when apparent motion was perceived between two fragmentary images, the observers could not tell, without close scrutiny, whether the two images were identical or complementary. A similar observation was made by Biederman and Cooper (1991b), who found that complementary images of common objects are subjectively experienced as equivalent, and that they require scrutiny before their differences become apparent.

In sum, the results of Experiment 3 suggest that apparent motion can establish correspondence between complementary views of planar objects as they are interpreted in 3-D space. This suggests that correspondence matching can be performed after a 3-D representation of a "restored" object has been generated (see Anstis \& Ramachandran, 1985; Shepard \& Judd, 1976; Shimojo \& Nakayama, 1990).

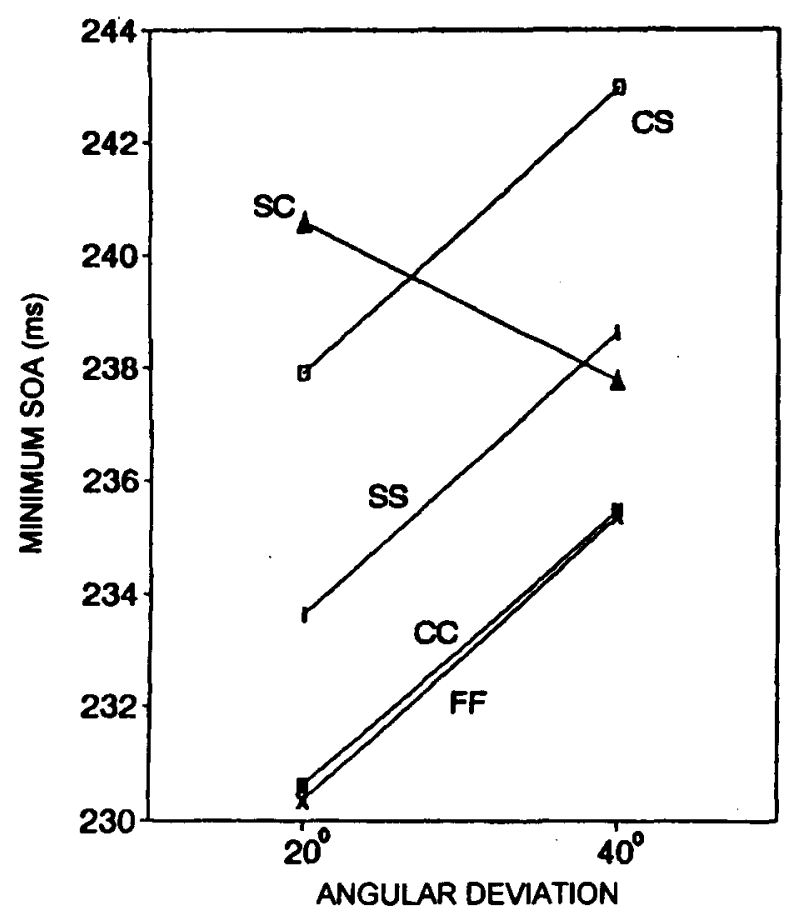

Figure 5. Mean minimum stimulus onset asynchrony as a function of angular deviation for the five pair types used in Experiment 3 (F, full; $\mathrm{S}$, sides; $\mathrm{C}$, corners).

\section{EXPERIMENT 4 \\ Apparent Rotational Motion in Depth of a 3-D Object}

In Experiment 3, 3-D motion of a 2-D object was examined; in Experiment 4, line drawings of a rectangular polyhedron (block) were used to examine 3-D motion of a 3-D object. The stimuli (A and B in Figure 6, bottom row) portray two complementary perspective views of a block. Like the stimuli used in Experiment 3, they are complementary in the sense that when properly aligned at the level of the apparent 3-D object representation, they create an intact perspective drawing of a block. The question was whether these figures would produce the same type of apparent rigid rotation in depth as that produced by their corresponding intact versions.

\section{Method}

Stimuli. The stimuli were the full, sides, and corners versions of two perspective views of a block. The top row of Figure 6 illustrates the three versions of one view, and the bottom row illustrates a complementary pair. Each member depicts a different view. The two views are related by a $10^{\circ}$ rotation about each of the three axes. The longest side of the block subtended $3.5 \mathrm{~cm}$.

Procedure. The experiment was divided into two parts. Part 1 consisted of one practice and one experimental block. Each block included five trials that represented a random ordering of the five pair types: FF, CC, SS, CS, and SC. Part 2 was exploratory, and it included four pairs in which only one member was fragmentary: $\mathrm{FS}, \mathrm{SF}, \mathrm{FC}$, and $\mathrm{CF}$, in a random order.

The subjects sat $80 \mathrm{~cm}$ from the screen so that the longest side of the block subtended $2.5^{\circ}$. Interframe interval was $100 \mathrm{msec}$, and stimulus duration was initialized at $60 \mathrm{msec}$.

Subjects. Ten University of Haifa students participated for course credit. They were not aware of the purpose of the experiment, and none of them had participated in the previous experiments.

\section{Results and Discussion}

For Part 1, SOAs averaged $254.7 \mathrm{msec}$ for FF, $277.7 \mathrm{msec}$ for SS, $250.0 \mathrm{msec}$ for CC, $287.9 \mathrm{msec}$ for $\mathrm{SC}$, and $307.6 \mathrm{msec}$ for CS. Overall, complementary pairs required longer mean SOAs $(297.8 \mathrm{msec} ; S E=$ $28.1 \mathrm{msec})$ than identical pairs $(260.8 \mathrm{msec} ; S E=$ $17.4 \mathrm{msec})[F(1,9)=5.88, p<.05]$, but they, too, clearly produced apparent motion in depth.

For Part 2, SOAs averaged $265.1 \mathrm{msec}$ for FS, $257.7 \mathrm{msec}$ for SF, $291.5 \mathrm{msec}$ for $\mathrm{FC}$, and $270.5 \mathrm{msec}$ for CF. A one-way ANOVA yielded $F<1$. Overall mean SOA for Part 2 (271.2 msec) was intermediate between that of the identical and the complementary pairs of Part 1, and it did not differ significantly from that for either of the pair types.

In sum, apparent motion in depth was found for the complementary versions of a 3-D object, requiring somewhat longer SOAs than the identical versions.

\section{EXPERIMENT 5} Subjective Ratings of Apparent Motion

Experiment 5 was designed primarily to convey to the reader some information about the goodness of apparent 
Full
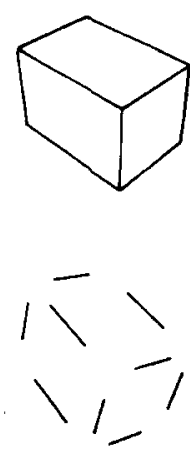

A
Sides
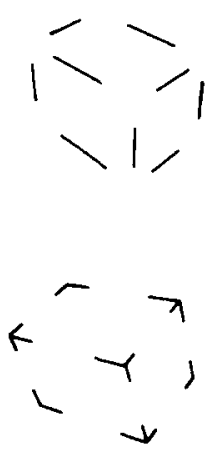

Figure 6. Stimuli used in Experiment 4 comprising two perspective views (A and B in bottom row). The top row illustrates the full, sides, and corners versions of perspective view $B$ only.

motion associated with complementary images of the same object, particularly in comparison with the apparent motion obtained between images depicting different objects. An important question is whether the motion perceived between two fragmentary images depends on whether or not they depict the same object. As noted earlier, previous studies, in which (intact) shapes that were related by a rotation transformation were used, yielded a systematic increase in minimum SOA with increased angular deviation between the shapes. This relationship suggested that apparent rotational motion is sensitive to figural properties (e.g., Farrell \& Shepard, 1981; Shepard \& Judd, 1976). However, there is sufficient evidence that apparent motion can also occur between objects that do not match in shape. Therefore, it is necessary to show that the kind of apparent motion observed between complementary images cannot be produced by two images that do not depict the same object. In Experiment 5, then, fragmentary pairs were included which in some cases depicted the same object, whereas in other cases the two images depicted two different objects.

A second question concerns the extent to which object-based apparent motion depends on the object's being readily recoverable from each of the two images. In all of the previous experiments, the complete object was readily recoverable from each of the images. To obtain some preliminary information regarding the importance of recoverability, Experiment 5 included highrecoverability (HR) fragmentary images of a rectangle as well as low-recoverability (LR) images, in which the rectangle was less readily recoverable from each image. The distinction between the HR and LR stimuli was made intuitively.

\section{Method}

Stimuli. The experiment included six pair types (see Figure 7). Three types involved identical shapes: full, fragmentary-HR, and
fragmentary-LR. Two additional types were complementary: one involved HR images (as in Experiment 1), and the other involved LR images, in which the complete rectangle was less readily perceived. The rectangles subtended $4.6 \times 2.3 \mathrm{~cm}$. Finally, the "different" pair consisted of fragmentary images of a square and a triangle; the sides in each image subtended $2.3 \mathrm{~cm}$.

Procedure. The subjects viewed the screen at a distance of $80 \mathrm{~cm}$. On each trial, two images were displayed in sequential alternation, for a total of eight frames, as in the previous experiments, so that odd-numbered frames always consisted of a $0^{\circ}$ angle (i.e., as in Figure 7). In the remaining frames, the image appeared $40^{\circ}, 60^{\circ}$, or $80^{\circ}$, rotated clockwise or counterclockwise. SOA was either 210 or $250 \mathrm{msec}$, and stimulus duration was $100 \mathrm{msec}$ in both cases.

The experiment included six blocks. Each block consisted of 36 trials each, which represented all combinations of pair type (6), angular deviation $\left( \pm 40^{\circ}, \pm 60^{\circ}, \pm 80^{\circ}\right.$ ), and order (i.e., which of the two images was the first in the sequence). Choice of rotation direction (clockwise or counterclockwise) was random. Also, because the two fragmentary pair types were each represented by two pairs of images (see Figure 7), the choice between them was determined randomly.

On each trial, the subjects saw the stimulus pair alternating. They were required to rate the goodness of the apparent motion on a 9-point scale, in which 1 designated very poor, and 9 designated very good. A "good" motion was defined as one in which a single object was perceived as moving smoothly from one position to another while preserving its overall shape. A "poor" motion was defined as one that was either jumpy and discontinuous, or one in which the object did not preserve its rigid shape when moving for example, when parts of the object jumped or twisted. The subjects initiated each trial by pressing the space bar. They were free to review the sequence as many times as they wished before making their ratings. The order of stimuli within each block was random, with the restriction that each set of six successive trials included one stimulus of each pair type.

In each block, only one 210 - or 250-msec SOA was used. SOAs were changed between blocks, but for each block they were counterbalanced across subjects. The experiment also included a practice block of 12 trials, in which 5-cm-long lines were used.

Subjects. Ten University of Haifa students participated for course credit. They were naive as to the purpose of the experiment. 


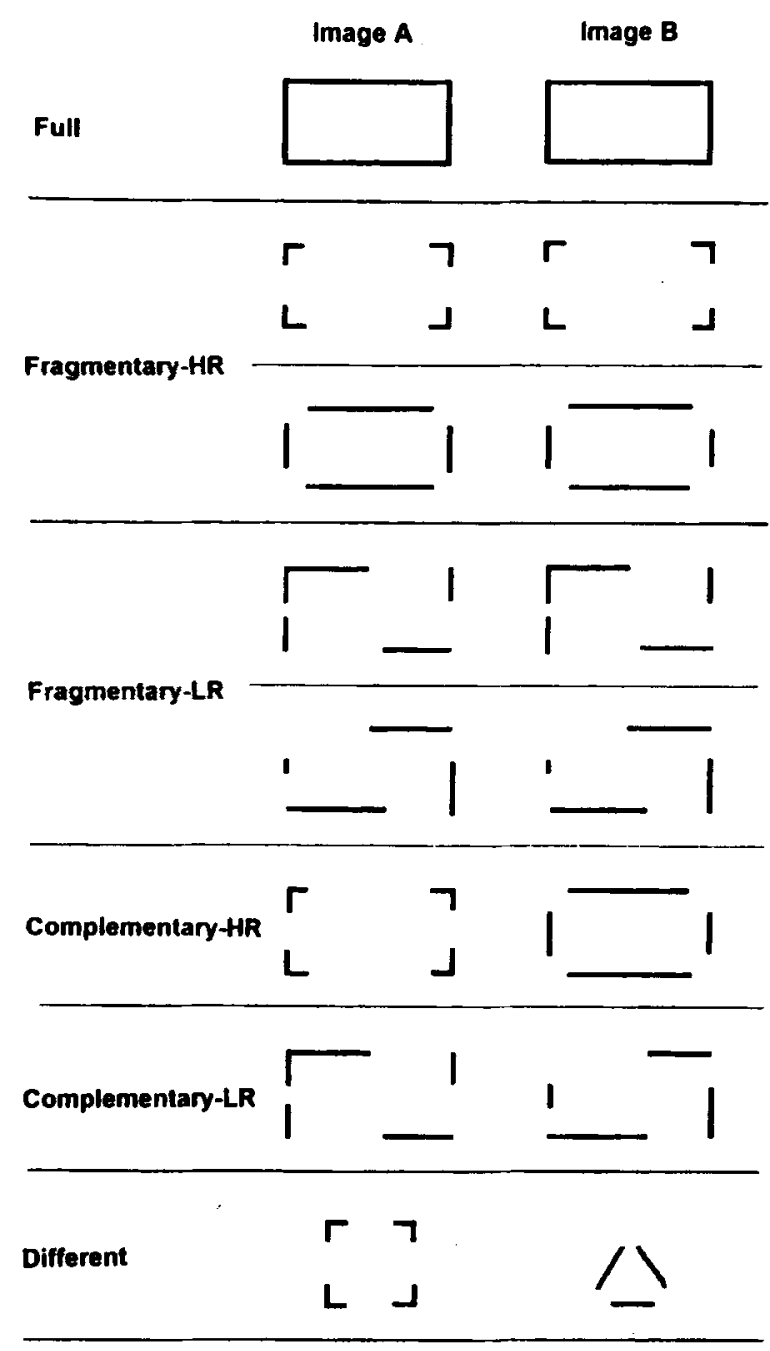

Figure 7. Stimuli used in Experiment 5.

\section{Results}

The first two blocks of the experiment served as practice, and they were not included in the following analyses. Preliminary analyses indicated a very similar pattern of results for both SOAs, so the results for both SOAs were collapsed. Figure 8 presents mean subjective ratings as a function of angular deviation for the six pair types. Overall, the highest ratings were assigned to the three pair types involving identical shapes. A two-way ANOVA (pair type $\times$ angular deviation) for these pairs yielded $F(2,18)=3.59, p<.05$ for pair type; $F(2,18)=$ $53.84, p<.0001$ for angular deviation; and $F(4,36)=$ 2.07 , n.s., for the interaction. The full pair yielded somewhat better apparent motion than the fragmentary pairs, but the quality of apparent motion improved systematically with decreasing angular separation for all of the pairs.

A two-way ANOVA that contrasted the HR and LR pairs yielded $F<1$ for pair type; $F(2,18)=7.14, p<.01$ for angular deviation; and $F(2,18)=1.23$, n.s., for the in- teraction. Thus, the complementary pairs also evidenced improved apparent motion with decreasing angular deviation, but the recoverability of the rectangle from its fragmentary images made no contribution to the quality of object-based apparent motion between them.

When the data across the two complementary pairs and the three identical pairs were collapsed, a two-way ANOVA of pair type (identical vs. complementary) $\times$ angular deviation yielded $F(1,9)=24.98, p<.001$ for pair type; $F(2,18)=29.23, p<.0001$ for angular deviation; and $F(2,18)=14.89, p<.001$ for the interaction. Thus, under the same conditions, identical pairs evidenced better apparent motion than complementary pairs, with the difference between them being most pronounced for small angular deviations.

Finally, the complementary pairs yielded considerably better apparent motion than the different pairs. A two-way ANOVA contrasting the complementary and different pairs yielded $F(1,9)=56.74, p<.0001$ for pair type; $F(2,18)=7.84, p<.01$ for angular deviation; and $F(2,18)=5.80, p<.05$ for the interaction. In fact, 7 of the subjects assigned a rating of 1 (very poor motion) to the "different" sequence throughout the entire experiment. Altogether, across all subjects and conditions, different pairs were assigned a rating of 1 in $95.0 \%$ of the cases, compared with $14.2 \%$ for complementary pairs and $2.6 \%$ for identical pairs.

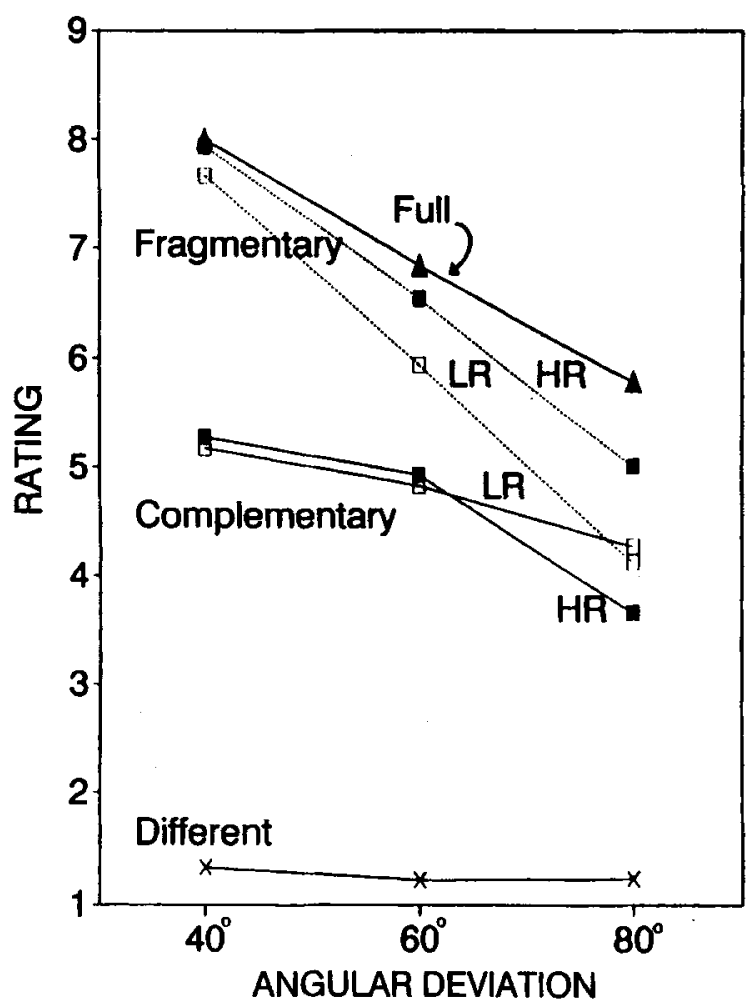

Figure 8. Mean ratings of the goodness of apparent motion as a function of angular deviation for the seven sequence types used in Experiment 5 (LR, low recoverability; HR, high recoverability). 


\section{Discussion}

The results of Experiment 5 indicated that complementary images of the same object produce apparent motion that differs in quality from that which may transpire between fragmentary images of different objects. It seems that a shape-preserving apparent motion is produced only when two fragmentary images can be seen as depicting the same object. Object-based apparent motion, then, seems to signal a process that is specifically concerned with the extraction of object representations.

The comparison between the LR and HR pairs failed to support the hypothesis that object-based apparent motion occurs only when the object can be readily recovered from the two images. However, the manipulation of recoverability may not have been adequate; in any case, it was not as strong as that achievable with images of real-life objects (see Biederman \& Cooper, 1991b). Note, though, that recent evidence suggests that, even with the latter type of objects, the amount of priming is about the same for both recoverable and nonrecoverable fragments (Srinivas, 1993).

Identical pairs produced better apparent motion than complementary pairs. Because completion is required for the interpretation of complementary images, it is possible that a longer SOA is needed to achieve apparent motion with a quality that is the same as that evidenced for the identical pairs. The observation that angular deviation exerted a stronger effect on identical pairs than it did on complementary pairs may indicate that the completion process and the correspondencematching process operate in parallel.

\section{GENERAL DISCUSSION}

The present study demonstrated that apparent motion can occur at the level of object representations between successive images that cannot be matched on the basis of their explicit visual features. Assuming a withinframe assembly process that helps to define one or more coherent units, apparent motion can then use the output of this process as the basis for cross-frame correspondence matching (see Cavanagh \& Mather, 1989). The present results do not deny that apparent motion is often based on local correspondence, and may be so even when such correspondence conflicts with within-frame organization (Anstis, 1970; Ullman, 1979). Rather they add more support to the claim that long-range apparent motion may also follow the extraction of form (Petersik, 1989; Ramachandran \& Anstis, 1986) and may be based on higher level, derived visual representations. What is interesting about the apparent motion produced by some of the complementary images (e.g., Figure 1) is that each visual segment in one image is, in fact, aligned with its missing (albeit inferred) partner in the other image. The experiments with complementary images in the depth plane further suggest that object-based apparent motion rests on correspondence at the level of the 3-D interpretation, rather than at the level of the projected
2-D image, so the 3-D interpretation must be formed before correspondence matching.

The apparent motion produced by complementary images accords with what Cavanagh and Mather (1989) designated as second-order motion. Such motion can respond to spatiotemporal correlations of texture or binocular disparity in the absence of any first-order correlation (e.g., Ramachandran et al., 1973). They have shown that the motion of a form can be perceived despite changes in the attributes that define it. According to $\mathrm{Ca}$ vanagh and Mather, the same mechanism underlies lower level and higher level motion, except that each responds to different stimulus properties. The observation that SOA tended to increase with angular disparity for both identical and complementary images of the present study may be construed as supporting this claim.

It is instructive to compare the results of the present study with those obtained with the priming technique. In general, the naming of an object is facilitated by the prior presentation of that object a few minutes earlier. Importantly, priming occurs even when different fragments of the object are presented at study and at test (Biederman \& Cooper, 1991a, 1991b; Cooper, Biederman, \& Hummel, 1992; Snodgrass \& Feenan, 1990; Srinivas, 1993). In Biederman and Cooper's (1991b) study, naming a contour-deleted image of an object was equally facilitated by the prior presentation of its complementary image as it was by a presentation of an identical image, but other studies have indicated stronger priming when the same images were used both at study and at test (Jacoby, Baker, \& Brooks, 1989; Snodgrass \& Feenan, 1990; Srinivas, 1993). The apparent-motion results of the present study are consistent with the latter findings; motion was perceived between two complementary images, but it generally required longer SOAs than when two identical images were used.

In Biederman's studies, the magnitude of the priming effect was also unaffected by several visual properties, including reflection and orientation. This suggests that priming is mediated by the activation of viewpointinvariant structural descriptions. Although Srinivas's (1993) recent findings indicate that priming is sensitive to viewpoint, significant transfer was also observed across two views of the same object. Thus, Srinivas concluded that priming is mediated by a structural description of objects. Apparent rotational motion, in contrast, seems to operate on viewpoint-specific representations. It is possible that object recognition is mediated by a visual representation that is different from that underlying the establishment of visual correspondence between events. Object naming seems to tap access to long-term object representations, whereas apparent motion entails comparison processes that are concerned with the extraction of invariance across episodic events (see Biederman \& Cooper, 1992; Cooper et al., 1992). Indeed, in a recent unpublished study, I found that backward alignment is also obtained between two complementary images of the same orientation-invariant shape. Backward alignment (see Koriat \& Norman, 1988, 1989; Ko- 
riat, Norman, \& Kimchi, 1991) is a process that transpires in the context of a mental rotation task, in which the response is based on bringing the current stimulus into alignment with the preceding stimulus, rather than on comparing it with its internal, canonical representation. Like apparent motion, backward alignment also appears to be concerned with the extraction of invariance across successive events.

Although the results of the present study were generally consistent across the five experiments, the experiments themselves were largely demonstrative in nature, documenting a phenomenon that apparently occurs under some circumscribed conditions. Several important questions remain open, and they should be pursued in future research. First, still unclear is the nature of the withinframe unit formation process that is assumed to precede cross-frame correspondence matching or to go hand in hand with it. Contour completion may be accomplished by visual routines that operate on the image features themselves, or it may be mediated by the activation of object/geon representations that help to assemble the visual fragments into coherent units, without the missing contours ever having to be restored (Biederman \& Cooper, 1991b; Blickle, 1989). In fact, in the apparent motion between two complementary images, the impression was that of a moving surface or body rather than the motion of outline figures.

Whatever the case, there is evidence suggesting that the processing of fragmentary images takes more time than the processing of intact, nonoccluded images (Biederman, 1987; Biederman \& Cooper, 1991b; Blickle, 1989; Sekuler \& Palmer, 1992). This might indicate either that the restoration/completion of fragmentary images takes time, or that objects/geons are more efficiently activated from intact than from contour-deleted images. Also, Enns and Rensink (1991) observed that preattentive processes cannot operate on contourdeleted images, and they depend on explicit connectivity of the line segments to afford rapid search. These observations may explain why SOAs for apparent motion were somewhat higher for complementary pairs than for identical pairs. The former pairs could be matched only at the level of the restored or recovered object representation, whereas the latter pairs could also be matched on the basis of the explicit visual features, before the images had been perceptually completed. If this interpretation is correct, correspondence matching ought to occur at the image and object levels in parallel, and image matching should be faster than object matching.

A second question pertains to the conditions under which object-based alignment can occur. As noted earlier, in the present study, a deliberate effort was made to use stimuli and procedures that favor the occurrence of objectbased apparent motion. It is critical, however, to specify the boundary conditions of this phenomenon. Several observations from exploratory work may be instructive.

First, apparent rotational motion has also been observed for complementary images of complex objects (e.g., an airplane similar to that depicted in Figure 22 in
Biederman, 1987). However, close scrutiny indicated that although the object as a whole was perceived as rotating, some of its parts were sometimes twisted or deformed, due to local alignments between noncorresponding fragments. Presumably, such accidental local alignments are less likely to occur when the intact images are used.

Note that in Experiments 1-4 of the present study, the complementary images were produced by having all edges in one image and all vertices in the other image. This was intended to rule out an explanation of the results in terms of apparent motion that is partly based on local matching. However, the results of Experiment 5 indicated that object-based apparent motion was no more likely to occur when each of the two images included a mixture of edges and vertices, and thus allowed more opportunities for competing local matches.

Second, apparent motion was not found with complementary areas of an enclosed shape (e.g., two black fragments of a triangular area, which, when aligned appropriately, created a black triangular area). This failure may be explained in terms of the proposition (Blickle, 1989; Nakayama et al., 1989) that fragmentary patches, such as Bregman's (1981) Bs and Leeper's (1935) stimuli, contain added misleading contours that prevent proper grouping and obstruct recognition. Blickle demonstrated that when the inappropriately added contours were removed from these stimuli, object recovery was much easier.

Third, apparent motion failed to recover a pattern that was not already perceived in each of the two images. Thus, the alternating display of two complementary images of a handwritten English word did not produce the impression of a rotating word when that word could not be identified from each of the individual images. This suggests that object-based apparent motion is obtained only with fragmentary figures that are recoverable (Biederman, 1987; Blickle, 1989). Although this suggestion may seem incompatible with the results of Experiment 5, it should be noted that, even in the LR fragmentary images of that experiment, the object (rectangle) could be perceived in each of the two images.

A final question concerns the underlying process. Cavanagh and Mather (1989) have detailed a model of second-order motion, and that model can be extended to account for the present findings by adding assumptions regarding the within-frame completion process. Another possibility that should be explored is that the type of object-based apparent motion observed in the present study is due to a process that constructs a sensible trajectory between two images after correspondence has occurred. Such a process may be sensitive to top-down activations, such as those that may be responsible for favoring the perception of motion along plausible biological trajectories (e.g., Shiffrar \& Freyd, 1990). If so, object-based apparent motion may be telling us less about the process by which correspondence itself is computed than about the process of interpreting such correspondence after it occurs. 
In conclusion, the apparent-motion results of the present study are consistent with the view that apparent motion occurs at a level that is concerned with the internal modeling of distal objects in the external world (Shepard \& Cooper, 1982). These results join with several recent trends in cognitive psychology that emphasize the role of ecologically relevant, object-centered representations in perceptual organization and attention (Baylis \& Driver, 1993; Enns \& Rensink, 1991; Farah, Brunn, Wong, Wallace, \& Carpenter, 1990; Kahneman, Treisman, \& Gibbs, 1992; Kanwisher \& Driver, 1992; Kramer \& Jacobson, 1991; Tipper, Driver, \& Weaver, 1991).

\section{REFERENCES}

ANSTIS, S. M. (1970). Phi movement as a subtraction process. Vision Research, 10, 1411-1430.

ANSTIS, S. M., \& RamachandRan, V. S. (1985). Kinetic occlusion by apparent movement. Perception, 14, 145-149.

AtTNEave, F., \& Block, G. (1973). Apparent motion in tridimensional space. Perception \& Psychophysics, 13, 301-307.

BAYLIS, G. C., \& DRIVER, J. (1993). Visual attention and objects: Evidence for hierarchical coding of location. Journal of Experimental Psychology: Human Perception \& Performance, 19, 451-470.

Biederman, I. (1987). Recognition-by-components: A theory of human image interpretation. Psychological Review, 94, 115-147.

Biederman, I., \& CoOPER, E. E. (1991a). Evidence for complete translational and reflectional invariance in visual object priming. Perception, 20, 585-593.

Biederman, I., \& COOPER, E. E. (1991b). Priming contour-deleted images: Evidence for intermediate representations in visual object recognition. Cognitive Psychology, 23, 393-419.

Biederman, I., \& COOPER, E. E. (1992). Size invariance in visual object priming. Journal of Experimental Psychology: Human Perception \& Performance, 18, 121-133.

BLICKLE, T. W. (1989). Recognition of contour deleted images. Unpublished doctoral dissertation, State University of New York at Buffalo.

BRADDICK, O. J. (1980). Low-level and high-level processes in apparent motion. Philosophical Transactions of the Royal Society of London: Series B, 290, 137-151.

BREGMAN, A. S. (1981). Asking the "what for" question in auditory perception. In M. Kubovy \& J. R. Pomerantz (Eds.), Perceptual organization (pp. 99-118). Hillsdale, NJ: Erlbaum.

Bundesen, C., LARSEN, A., \& FARRELl, J. E. (1983). Visual apparent movement: Transformations of size and orientation. Perception, 12 , 549-558.

Cavanagh, P., \& Mather, G. (1989). Motion: The long and the short of it. Spatial Vision, 4, 103-129.

Cooper, E. E., Biederman, I., \& Hummel, J. E. (1992). Metric invariance in object recognition: A review and further evidence. Canadian Journal of Psychology, 46, 191-214.

Corbin, H. H. (1942). The perception of grouping and apparent movement in visual depth. Archives of Psychology, 273, 1-50.

EnNS, J. T., \& REnsink, R. A. (1991). Preattentive recovery of threedimensional orientation from line drawings. Psychological Review, 98, 335-351.

Farah, M. J., BrunN, J. L., Wong, A. B., Wallace, M. A., \& CarPENTER, P. A. (1990). Frames of reference for allocating attention: Evidence from the neglect syndrome. Neuropsychologia, 28, 335347.

FARRELL, J. E. (1983). Visual transformations underlying apparent movement. Perception \& Psychophysics, 33, 85-92.

Farrell, J. E., Larsen, A., \& Bundesen, C. (1982). Velocity constraints on apparent rotational movement. Perception, 11, 541-546.

Farrell, J. E., \& ShEPard, R. N. (1981). Shape, orientation, and apparent rotational motion. Journal of Experimental Psychology: Human Perception \& Performance, 7, 477-486.
Green, M., \& ODom, J. V. (1986). Correspondence matching in apparent motion: Evidence for three-dimensional spatial representation. Science, 233, 1427-1429.

Grossberg, S., \& Mingolla, E. (1985). Neural dynamics of perceptual grouping: Textures, boundaries, and emergent segmentations. Perception \& Psychophysics, 38, 141-171.

HuMmel, J. E., \& Biederman, I. (1992). Dynamic binding in a neural network for shape recognition. Psychological Review, 99, 480-517.

JACOBY, L. L., BAKER, J. G., \& BROOKS, L. R. (1989). Episodic effects on picture identification: Implications for theories of concept learning and theories of memory. Journal of Experimental Psychology: General, 118, 306-340.

Kahneman, D., Treisman, A., \& GibBs, B. J. (1992). The reviewing of object files: Object-specific integration of information. Cognitive Psychology, 24, 175-219.

KanIZsa, G. (1979). Organization in vision: Essays on Gestalt perception. New York: Praeger.

KANWISHer, N., \& DrIver, J. (1992). Objects, attributes, and visual attention: Which, what, and where. Current Directions in Psychology, 1, 26-31.

KellmaN, P. J., \& Shipley, T. F. (1991). A theory of visual interpolation in object perception. Cognitive Psychology, 23, 141-221.

KLEFFNER, D. A., \& RAMACHANDRAN, V. S. (1992). On the perception of shape from shading. Perception \& Psychophysics, 52, 18-36.

KoFFKA, K. (1935). Principles of Gestalt psychology. New York: Harcourt, Brace \& World.

Kolers, P. A. (1972). Aspects of motion perception. New York: Pergamon.

Kolers, P. A., \& Pomerantz, J. R. (1971). Figural change in apparent motion. Journal of Experimental Psychology, 87, 99-108.

Koriat, A., \& Norman, J. (1988). Frames and images: Sequential effects in mental rotation. Journal of Experimental Psychology: Learning, Memory, \& Cognition, 14, 93-111.

Koriat, A., \& NORMAN, J. (1989). Establishing global and local correspondence between successive stimuli: The holistic nature of backward alignment. Journal of Experimental Psychology: Learning, Memory, \& Cognition, 15, 480-494.

KorIaT, A., NoRMAN, J., \& KIMCHI, R. (1991). Recognition of rotated letters: Extracting invariance across successive and simultaneous stimuli. Journal of Experimental Psychology: Human Perception \& Performance, 17, 444-457.

KORTE, A. (1915). Kinematoskopische Untersuchungen [Kinematoscopic investigations]. Zeitschrift für Psychologie, 72, 194-296.

Kramer, A. F, \& JaCOBSON, A. (1991). Perceptual organization and focused attention: The role of objects and proximity in visual processing. Perception \& Psychophysics, 50, 267-284.

LEEPER, R. (1935). A study of a neglected portion of the field of learning: The development of sensory organization. Journal of Genetic Psychology, 46, 41-75.

MATHER, G. (1988). Temporal properties of apparent motion in subjective figures. Perception, 17, 729-736.

MCBEATH, M. K., \& SHEPARD, R. N. (1989). Apparent motion between shapes differing in location and orientation: A window technique for estimating path curvature. Perception \& Psychophysics, 46, 333-337.

MuTch, K., SMITH, I. M., \& YoNAS, A. (1983). The effect of twodimensional and three-dimensional distance on apparent motion. Perception, 12, 305-312.

Nakayama, K., Shimojo, S., \& Silverman, G. H. (1989). Stereoscopic depth: Its relation to image segmentation, grouping, and the recognition of occluded objects. Perception, 18, 55-68.

PETERSIK, J. T. (1989). The two-process distinction in apparent motion. Psychological Bulletin, 106, 107-127.

PitTenger, J. B., \& SHAw, R. E. (1977). Comment on Warren's Visual information for object identity in apparent movement. Perception \& Psychophysics, 22, 104-105.

RAMACHANDRAN, V. S. (1990). Interactions between motion, depth, color and form: The utilitarian theory of perception. In C. Blakemore (Ed.), Vision: Coding and efficiency (pp. 346-360). Cambridge: Cambridge University Press.

RamaCHANDRAN, V. S., \& ANSTIS, S. M. (1986). Figure-ground segregation modulates apparent motion. Vision Research, 26, 19691975. 
Ramachandran, V. S., RaO, V. M., \& Vidyasagar, T. R. (1973). Apparent movement with subjective contours. Vision Research, 13, 1399-1401.

Sekuler, A. B., \& Palmer, S. E. (1992). Perception of partly occluded objects: A microgenetic analysis. Journal of Experimental Psychology: General, 121, 95-111.

SHEPARD, R. N. (1984). Ecological constraints on internal representation: Resonant kinematics of perceiving, imagining, thinking, and dreaming. Psychological Review, 91, 417-447.

ShePARD, R. N., \& COOPER, L. A. (1982). Mental images and their transformations. Cambridge, MA: MIT Press/Bradford Books.

ShePaRd, R. N., \& JudD, S. A. (1976). Perceptual illusion of rotation of three-dimensional objects. Science, 191, 952-954.

ShIfFRAR, M., \& FREYD, J. J. (1990). Apparent motion of the human body. Psychological Science, 1, 257-264.

Shimojo, S., \& NaKayama, K. (1990). Amodal representation of occluded surfaces: Role of invisible stimuli in apparent motion correspondence. Perception, 19, 285-299.

Shimojo, S., Silverman, G. H., \& Nakayama, K. (1989). Occlusion and the solution to the aperture problem for motion. Vision Research, 29, 619-626.

Snodgrass, J. G., \& Feenan, K. (1990). Priming effects in picture fragment completion: Support for the perceptual closure hypothesis. Journal of Experimental Psychology: General, 119, 276296.

SRINIVAS, K. (1993). Perceptual specificity in nonverbal priming. Jour- nal of Experimental Psychology: Learning, Memory, \& Cognition, 19, 582-602.

TIPPER, S. P., Driver, J., \& WeAver, B. (1991). Object-centered inhibition of return of visual attention. Quarterly Journal of Psychology, 43A, 289-298.

UlLMAN, S. (1978). Two dimensionality of the correspondence process in apparent motion. Perception, 7, 683-693.

Ullman, S. (1979). The interpretation of visual motion. Cambridge, MA: MIT Press.

VICTOR, J. D., \& CONTE, M. M. (1990). Motion mechanisms have only limited access to form information. Vision Research, 30, 289-301.

WARREN, W. H. (1978). Visual information for object identity in apparent movement. Perception \& Psychophysics, 21, 264-268.

WARREN, W. H., \& Shaw, R. E. (1978). The visual specification of events: A response to Ullman. Perception \& Psychophysics, 24, 387-389.

\section{NOTE}

1. The stimuli were presented on a color monitor with a refresh rate of $72 \mathrm{~Hz}$, so SOA could be controlled down only to about $14 \mathrm{msec}$. Therefore, at the initial SOA settings, the increments were only approximated.

(Manuscript received August 16, 1993; revision accepted for publication April 11, 1994.) 